\title{
A Case of Stent Strut Fracture of a Paclitaxel-Eluting Stent at the Time of Stent Implantation in a Complex Coronary Lesion
}

\author{
Hyun-Sook Lee, RN, Seung-Ho Hur, MD, Chang-Wook Nam, MD, Yun-Kyeong Cho, MD, \\ Hyungseop Kim, MD, Seong-Wook Han, MD, Kwon-Bae Kim, MD and Yoon-Nyun Kim, MD \\ Division of Cardiology, Department of Internal Medicine, Keimyung University Dongsan Medical Center, Daegu, Korea
}

\section{ABSTRACT}

Several cases of stent strut fractures (SSFs) have recently been reported following drug-eluting stent (DES) implantation Although SSF is a rare complication, it has been suggested to be a cause of restenosis. To date, a number of cases of stent fracture have been associated with sirolimus-eluting stents and they occurred from 2 days to about 2 years after the initial procedure. We report here on a case of paclitaxel-eluting stent fracture at the time of stent placement in a calcified coronary lesion. (Korean Circ J 2008;38:387-389)

KEY WORDS: Stent, fracture; Drug-eluting stents.

\section{Introduction}

Treatment of de novo coronary artery lesions with drug-eluting stent (DES) has led to a marked reduction in the restenosis rates. However, restenosis post-DES implantation continues to be a challenge, and particularly in the setting of diabetes, multiple stent use and small-vessel disease. ${ }^{1)}$ Stent strut fractures (SSF) have been identified during follow-up angiographic and intravascular ultrasound (IVUS) examinations and this appears to be a predictor of restenosis after DES implantation. Although all the reported SSFs were detected within a few days to several months after stent implantation, ${ }^{2-5)}$ no SSF has been thus far reported during the initial procedure. We report here on a case of SSF that occurred during the index procedure in a calcified coronary lesion.

\section{Case}

A 74-year-old man was admitted with angina pectoris and he had suffered from typical chest pain for the previous 1 month. The initial electrocardiogram (ECG) exhibited left ventricular hypertrophy and no ischemic

Received: February 26, 2008

Accepted: March 24, 2008

Correspondence: Seung-Ho Hur, MD, Division of Cardiology, Department of Internal Medicine, Keimyung University Dongsan Medical Center, 194 Dongsan-dong, Jung-gu, Daegu 700-712, Korea

Tel: 82-53-250-7949, Fax: 82-53-250-7034

E-mail:shur@dsmc.or.kr changes. Among the known cardiovascular risk factors, the patient had a 7-year history of hypertension and he was a current smoker. Transthoracic echocardiography showed an ejection fraction of $65 \%$ and no regional wall motion abnormalities. The coronary angiogram revealed triple-vessel coronary artery disease, including significant stenosis of the mid left anterior descending (LAD), left circumflex (LCX), and right coronary (RCA) arteries (Fig. 1 ). The patient underwent percutaneous coronary intervention (PCI) of the mid RCA with employing a transradial approach. With the support of a $6 \mathrm{~F}$ RB guiding catheter (Radiobrachia; Cordis, Miami Lakes, FL, USA), a Runthrough NS guidewire (Terumo, Tokyo, Japan) was easily placed into the distal RCA. After predilation with a $2.5 \times 20 \mathrm{~mm}$ Sprinter balloon (Medtronic, Minneapolis, MN, U.S.A), an IVUS (Atlantis SR Pro $40 \mathrm{MHz}$; Boston Scientific, Natick, MA, U.S.A) was performed to evaluate the geometry and characteristics of the plaque at the target lesion. There was a significant plaque burden with eccentric intraluminal calcium deposits [calcium degree: $96^{\circ}$, minimal lumen area (MLA) $1.73 \mathrm{~mm}^{2}$, plaque burden: $86 \%$ ]. Based on these IVUS findings, a 4.0 $\times 20 \mathrm{~mm}$ Paclitaxel-Eluting Stent (PES; Taxus Express 2, Boston Scientific, Natick, MA, U.S.A) was implanted at the mid RCA with using 14 atmospheres (atm) of pressure. Residual stenosis was seen in the middle of the RCA during coronary angiogram, and the subsequent IVUS examination showed stent underexpansion as well as asymmetric stent expansion due to the eccentric calcified lesion. During pullback of the IVUS catheter, few or no stent struts were observed on the cross-sectional 
images at the transional zone where the eccentric calcified lesion with the adjacent minimal atherosclerotic plaque was located. To improve the stent underexpansion, an adjunctive $4.0 \times 12 \mathrm{~mm}$ Quantum Maverick balloon (Boston Scientific, Maple Grove, MN, U.S.A) was inflated using $14 \mathrm{~atm}$ of pressure; after which, the final IVUS revealed that the gap of the SSF at the transional zone was wider than that following the post-stent IVUS examination, with an increase of the minimal stent area from 6.25 to $7.10 \mathrm{~mm}^{2}$ (Fig. 2). Although the SSF occurred at the proximal stented segment, the coronary angiogram continued to show thrombolysis in myocardial infarction (TIMI) 3 flow with minimal residual stenosis. There was no procedure-related complication during the hospital stay. The patient was discharged with a prescription for indefinite triple antiplatelet therapy (aspirin 100 mg, clopidogrel $75 \mathrm{mg}$ and cilostazol $200 \mathrm{mg}$ ). During 9 months of clinical follow-up, there were no adverse

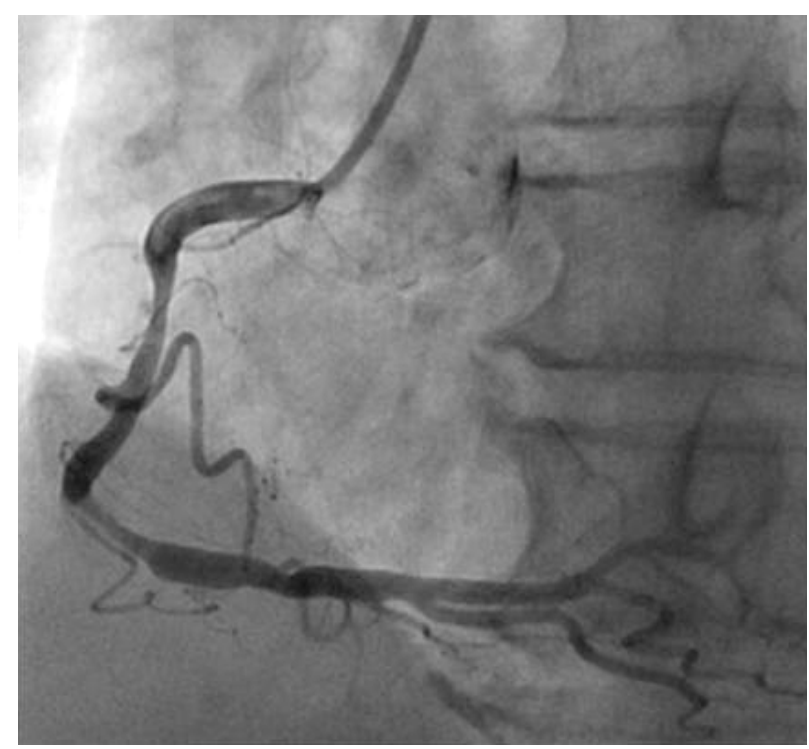

Fig. 1. Initial angiogram of the right coronary artery (RCA) before the procedure. Severe stenosis was observed in the mid portion of the RCA. cardiac events and the follow-up coronary angiogram showed TIMI 3 flow with minimal stenosis. There was no evidence of restenosis at the site of the SSF in the mid RCA (Fig. 3).

\section{Discussion}

Stent strut fracture is defined as interruption and/or an uncovered stent strut area by performing quantitative coronary angiography (QCA) and there are none or fewer visible stent struts on the cross-sectional view by performing IVUS within the stented segments. ${ }^{5)}$ A recent report has suggested 3 patterns of SSF based on the QCA findings: disruption, avulsion and displacement. ${ }^{5)}$ The incidence of SSF has been reported to be $1 \%$ to $3 \%$ of all DES implantation cases. ${ }^{5-7)}$ Although $\mathrm{SSF}$ is a rare complication in the DES era, this phenomenon may be clinically important because of its potential

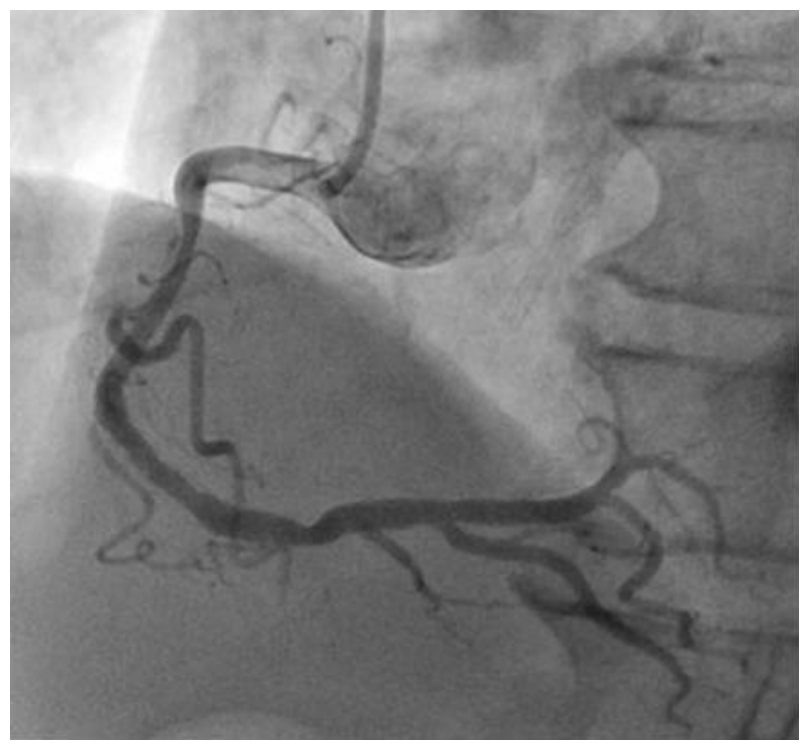

Fig. 3. Follow-up angiogram after 9 months post index procedure. Angiogram showed TIMI 3 flow with minimal stenosis within stented segments.
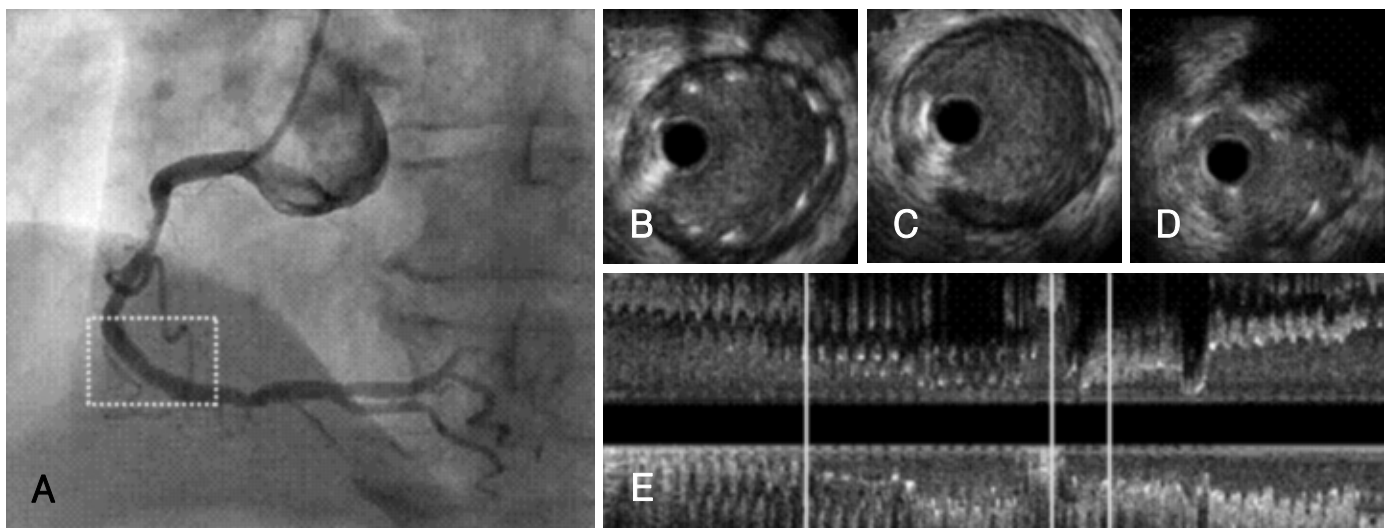

Fig. 2. Intravascular ultrasound image of the stent fracture site. Post-Stenting with (A) $4.0 \times 20 \mathrm{~mm}$. Paclitaxel-Eluting Stent Angiogram after stenting. Residual stenosis was seen in the middle of the RCA. (B) IVUS finding at proximal stent edge site. (C) Absence of stent strut in cross-sectional images at the transitional zone between eccentrically calcified lesion and mild atherosclerotic plaque lesion. (D) IVUS finding at the eccentrically calcified site. (E) Longitudinal view. 
association with clinical DES failure, including restenosis or target-lesion revascularization. ${ }^{5-7)}$ Interestingly, most of the reported SSFs have occurred in patients who were treated with sirolimus-eluting stents (SESs), ${ }^{45)}$ while thus far there has been only one reported case of SSF with restenosis 6-months after the procedure in a patient treated with $\mathrm{PES},{ }^{8)}$ This difference might be explained by the different cell designs between the SES and the $\mathrm{PES}^{9)}$ or simply by the different frequency of using these stents, among other reasons.

The PES has an open-cell design while the SES consists of a closed-cell design with a stainless steel platform. The open-cell design is expected to increase flexibility and deliverability and enhance side-branch access by staggering the cross-linking elements, thereby allowing better conformability on angulated anatomy; however, the external side of angled lesions can be excessively opened. On the other hand, the closed-cell design with links connecting the cell is expected to be associated with more uniform drug delivery throughout the entire target lesion area and to have a lesser tendency for plaque prolapse. ${ }^{12)}$ However, the connecting links are weaker than the main cell frame and they might be more easily fractured if the stent receives excessive stress such as occurs in highly tortuous anatomy or due to the vigorous bending by the beating heart. The relevance of the closed or open stent cell design on the likelihood of stent fracture remains to be determined.

Although the timing of SSFs remains unknown, recent studies have shown that SSFs mainly occur several months after the index procedure. ${ }^{45)}$ However, Kang et $\mathrm{al}^{3)}$ reported a case of SSF that occurred within 2 days after SES implantation. In our case, the SSF occurred at the time of stent implantation for the treatment of a calcified lesion, suggesting that SSFs can occur during stent implantation as well as during long-term follow-up.

Several studies have demonstrated that SSFs are more likely to occur in longer stents, at the overlapping sites of stents, in the RCA or in saphenous vein grafts, in very tortuous or highly moving vessel, in calcified lesion and at the inflated site with using a larger balloon or high pressure. ${ }^{57710) 11)}$ These circumstances are associated with excessive mechanical forces during cardiac contraction or with procedures that result in SSFs after DES implantation. Despite the relatively short lesion length in the case described in our report, an eccentric calcified lesion with adjacent minimal plaque in the middle angulated portion of the RCA may render a DES susceptible to SSF following implantation. Furthermore, severe stent underexpansion was observed at the calcified lesion (a minimal stent area) during the IVUS examination following DES implantation. Performing adjunctive balloon inflation with high pressure may have produced an uneven distribution of radial force between the minimal stent area and the adjacent well-expanded stent area, and this provoked the SSF in our case. Therefore, one could speculate that a biodegradable DES or DES with a more flexible stent platform might overcome this phenomenon after DES implantation.

The therapeutic strategy for in-stent restenosis that's driven by SSF is not well established. Generally, a PCI procedure is not recommended for a minimal amount of neointimal hyperplasia (NIH) that is without clinical symptomatology; however, a significant amount of NIH accompanied by clinical symptomatology is likely to be treated with either the same kind of DES used for the index procedure or with another kind of DES. Other treatment modalities for the case of focal restenosis within the DES area include plain old-style balloon angioplasty or stenting with a bare-metal stent. No further intervention was performed in this case because the 9month follow-up coronary angiogram showed minimal lumen narrowing at the suspected SSF site and the patient remained symptom-free.

\section{REFERENCES}

1) Hong SJ, Kim MH, Ahn TH, et al. Multiple predictors of coronary restenosis after drug-eluting stent implantation in patients with diabetes. Heart 2006;92:1119-24.

2) Kim EJ, Rha SW, Wani SP, et al. Coronary stent fracture and restenosis in the drug-eluting stent era: do we have clues of management? Int J Cardiol 2007;120:417-9.

3) Kang WY, Kim W, Kim HG, Kim W. Drug-eluting stent fracture occurred within 2 days after stent implantation. Int J Cardiol 2007;120:273-5.

4) Lee SH, Park JS, Shin DG, et al. Frequency of stent fracture as a cause of coronary restenosis after sirolimus-eluting stent implantation. Am J Cardiol 2007;100:627-30.

5) Chung WS, Park CS, Seung KB, et al. The incidence and clinical impact of stent strut fractures developed after drug-eluting stent implantation. Int J Cardiol 2008;125:325-31.

6) Lee MS, Jurewitz D, Aragon J, Forrester J, Makkar RR, Kar S. Stent fracture associated with drug-eluting stents: clinical characteristics and implications. Catheter Cardiovasc Interv 2007;69: 387-94.

7) Aoki J, Nakazawa G, Tanabe K, et al. Incidence and clinical impact of coronary stent fracture after sirolimus-eluting stent implantation. Catheter Cardiovasc Interv 2007;69:380-6.

8) Hamilos MI, Papafaklis MI, Ligthart JM, Serruys PW, Sianos G. Stent fracture and restenosis of a paclitaxel-eluting stent. Hellenic J Cardiol 2005;46:439-42.

9) Suzuki Y, Ikeno F, Yeung AC. Drug-eluting stent strut distribution: a comparison between Cypher and Taxus by optical coherence tomography. J Invasive Cardiol 2006;18:111-4.

10) Chowdhury PS, Ramos RG. Images in clinical medicine: coronarystent fracture. N Engl J Med 2002;347:581.

11) Sianos G, Hofma S, Ligthart JM, et al. Stent fracture and restenosis in the drug-eluting stent era. Catheter Cardiovasc Interv 2004;61:111-6.

12) Kim JS, Yoon YW, Hong BK, et al. Delayed stent fracture after successful sirolimus-eluting stent (Cypher ${ }^{\mathbb{B}}$ ) implantation. Korean Circ J 2006;36:443-9. 